\title{
Medizin, Recht und das Wissen vom «zivilisierten» Krieg im langen 19. Jahrhundert
}

Svenja Goltermann

\section{Summary}

The aim to 'civilize' warfare accompanied Medicine and International Law ever since the mid-19th century. However, the entanglement of Medicine and Law, crucial for such an endeavour, has not been taken into consideration so far; likewise, the huge importance of medical knowledge for the perception of wars and their ramifications did not garner much attention in historical research. Hence, by focusing on the 'long' $19^{\text {th }}$ century, this paper shows, firstly, that the production of surgical knowledge during warfare aimed at measuring the effects of combat on human bodies in order to develop prognostic medical knowledge for future wars, as well as maintaining the combat strength of soldiers. Moreover, this knowledge production during warfare strived for the enhancement of medical competence in the diagnosis and treatment of wounds in general. Secondly, I show that this medical knowledge was not only relevant for warfare, but also crucial for the design of International Law: it served to nourish the debates among the so called 'civilized' nations about legitimate and illegitimate weaponry and warfare.

\section{Zusammenfassung}

Das Bestreben, den Krieg zu «zivilisieren», begleitete die Medizin und das Völkerrecht seit der Mitte des 19. Jahrhunderts. Die dafür entscheidende Verschränkung von Medizin und Recht ist bislang allerdings unberücksichtigt geblieben; kaum beleuchtet in der historischen Forschung wurde auch die in diesem Zusammenhang grosse Bedeutung des medizinischen Wissens für

Svenja Goltermann, Universität Zürich, Historisches Seminar, Rämistrasse 64, 8001 Zürich (svenja.goltermann@fsw.uzh.ch) 
die Wahrnehmung von Kriegen und ihren Folgen. Dieser Aufsatz zeigt mit Blick auf das lange 19. Jahrhundert erstens, dass die Generierung von namentlich chirurgischem Wissen in Kriegen darauf zielte, deren Auswirkungen auf den Menschen exakter zu bestimmen, um prognostisches Wissen über medizinische Aspekte zukünftiger Kriegsverläufe entwickeln zu können und die Kampfkraft der Soldaten zu erhalten. Zugleich sollte es auch dazu dienen, die ärztliche Kompetenz zur Diagnose und Therapie von Wunden zu steigern. Zweitens geht es darum, deutlich zu machen, wie dieses kriegsrelevante medizinische Wissen in die völkerrechtlichen Debatten der so genannten «zivilisierten» Nationen über die Grenzen legitimer Kriegsführung einfloss.

Ein Jahr nach Beginn des Ersten Weltkrieges erschien in einer medizinischen Fachzeitschrift ein Artikel unter dem Titel «Eindrücke aus deutschen Kriegslazaretten». «Der gegenwärtige Weltkrieg» sei, hieß es darin, «für schon tüchtig vorgebildete Mediziner und Pflegerinnen» eine «hohe Schule für den Unterricht in modernster Kriegschirurgie», wie diese «für absehbare Zeit kaum mehr geboten sein dürfte». ${ }^{1}$ Der Schweizer Chirurg Theodor Kocher, Autor dieser Zeilen, war nicht der einzige, dem der Erste Weltkrieg wie ein großes Versprechen für die Verbesserung ärztlichen Könnens erschien. ${ }^{2}$ Selbst nach diesem Krieg, der Millionen von Menschen das Leben kostete und weitere Millionen als körperlich oder psychisch Versehrte hinterließ, rühmten Ärzte die medizinischen Fortschritte, die dieser Krieg hervorgebracht habe und die nun auf die zivile Welt übertragen werden könnten. ${ }^{3}$

Dass Kriege der Medizin zu einem Fortschritt verhelfen würden, ist ein Narrativ, das nicht nur aus dem Kontext des Ersten Weltkriegs bekannt ist. Diverse Studien haben es mittlerweile kritisch befragt - auch im Hinblick auf seine Herkünfte und Konsequenzen. ${ }^{4}$ Doch der Zusammenhang zwischen Krieg und medizinischer Wissensproduktion ist deshalb alles andere als hinreichend behandelt worden. Das gilt vor allem für die Frage nach der Bedeutung medizinischen Wissens für die Wahrnehmung von Kriegen und ihrer Folgen, die bislang bestenfalls am Rande berührt worden ist. Gänzlich außen vor blieb sogar die Verschränkung von Medizin und Recht - ein Phänomen, das in den vergangenen Jahrzehnten aufgrund der zunehmenden Bedeutung forensischer Untersuchungen im Kontext von Kriegs- und Menschenrechts-

1 Kocher 1915, 449-479, 478.

2 Vgl. u.a. Eckart 22003, 299-320, 300.

3 Vgl. u.a. von Schjerning 1920.

4 Vgl. dazu u.a. Berger 2009; Linton, 2000, 291-316; Schlich 2012, 308-334. 
verbrechen vermutlich präsenter ist, wobei es auch für diesen Zeitraum an Studien fehlt.

Im Folgenden sollen beide Aspekte - die Bedeutung medizinischen Wissens für die Wahrnehmung von Kriegen und die Verknüpfung von Medizin und Recht - beleuchtet werden, und zwar mit Blick auf das lange 19. Jahrhundert und mit weitgehender Beschränkung auf die Chirurgie. Dabei geht es vor allem um Praktiken der Wissensgenerierung, die zum einen darauf zielten, die Auswirkungen von Kriegen auf den Menschen exakter bestimmen und prognostisches Wissen über medizinische Aspekte zukünftiger Kriegsverläufe entwickeln zu können; zum andern hofften die Ärzte in Kriegen diagnostische und therapeutische Kompetenz zur Behandlung von Wunden zu erlangen, die sowohl in Kriegen als auch im zivilen Leben einsetzbar sein sollten. Diese Praktiken der Wissensgenerierung lassen sich in zahlreichen wissenschaftlichen Abhandlungen, die Chirurgen seit Mitte des 19. Jahrhunderts in zunehmender Zahl publizierten, erfassen. $\mathrm{Zu}$ diesen Praktiken gehörte die systematische Sammlung von Daten, die in Statistiken über Verletzungs- und Todesraten überführt wurden; sie stehen im ersten Abschnitt dieses Artikels zum Thema «Leben und Sterben erfassen» im Vordergrund. Weitere Praktiken, wie etwa die Anfertigung von Zeichnungen, von Fotografien oder auch das Experiment werden im nachfolgenden zweiten Teil «Experimentieren und regulieren» thematisiert. Dieser Abschnitt konzentriert sich auf die zweite Hälfte des langen 19. Jahrhunderts, in der verschiedene Bestimmungen des humanitären Völkerrechts darauf ausgerichtet waren, den Krieg zu «humanisieren» und zu «zivilisieren» ${ }^{5}$ - so die seinerzeit verwendeten Begriffe. Für diese Bestrebungen gab es viele Motive, darunter finanzielle und militärische, also keineswegs nur humanitäre. ${ }^{6}$ In diesem Artikel geht es mir aber darum, exemplarisch deutlich zu machen, auf welche Weise die völkerrechtlichen Regelungen und die Medizin miteinander verflochten waren. Wie das Völkerrecht auch, stellte die Medizin nämlich ein «Wissen» über den «zivilisierten» Krieg her. Zwei Punkte sollen hier angeschnitten werden: Zum einen produzierten Ärzte mithilfe von Versuchen kriegsrelevantes Wissen, das in die Ausgestaltung völkerrechtlicher Regelungen einging. Zum andern war die Medizin daran beteiligt, die Reichweite und die Grenzen des «zivilisierten» Krieges zu bestimmen. Dabei kamen Vorstellungen vom «unzivilisierten» Anderen zum Tragen, die dem

5 Vgl. u.a. Best 1980; Segesser 2007.

6 Vgl. u.a. Hutchinson 1997; Dülffer 2008, 77-88, die darauf verwiesen haben, dass es etwa für die Regierungsvertreter und Militärs durchaus ganz pragmatische Gründe, sowohl finanzieller als auch militärischer Art, gab, an der Verabschiedung der Konventionen mitzuwirken. 
Völkerrecht aufgrund seiner Bestimmung des «Zivilisierten» inhärent waren, ${ }^{7}$ an deren Herstellung jedoch auch die Medizin Anteil hatte.

\section{Leben und Sterben erfassen}

Sowohl in Europa als auch in den USA konnte Ende des 18. Jahrhunderts keine Rede davon sein, dass sich Ärzte damit befasst hätten, den Gesundheitszustand oder den Tod von Soldaten systematisch zu erfassen. Der Anstoß für eine zuverlässige Sicherstellung von Daten über die Mortalitätsraten im Militär ging auch nicht einmal von den dort tätigen Ärzten aus. Eine zentrale Rolle spielte vielmehr zunächst der Staat, der in einer Reihe von europäischen Ländern seit der Wende vom 18. zum 19. Jahrhundert ein gesteigertes Interesse an Information über den Gesundheitszustand seiner Soldaten entwickelte. Das Interesse, die militärische Stärke der Armee auch in Kriegszeiten zuverlässiger bestimmen zu können, war einer der Gründe dafür; ${ }^{8}$ ein anderer aber war die Neuregelung von Erbschaftsangelegenheiten, die den Hinterbliebenen veränderte Möglichkeiten eröffnete, einen Anspruch auf die Hinterlassenschaft eines im Krieg verstorbenen Soldaten zu übernehmen. ${ }^{9}$ Eine dafür unerlässliche Voraussetzung war jedoch die Feststellung seines Todes, wie auch die namentliche Identifizierung des Toten unabdinglich war. ${ }^{10}$ Doch die Verfahren, Namen und persönliche Daten von verstorbenen Soldaten zu sammeln, waren während der Revolutions- und Napoleonischen Kriege des späten 18. und frühen 19. Jahrhunderts völlig unzureichend, um den Anfragen von Angehörigen vermisster Soldaten nachzukommen. Die Folge waren offizielle Anordnungen von Seiten staatlicher Behörden, die Ärzten in Lazaretten vorgaben, welche Daten auf den Totenscheinen verstorbener Soldaten einzutragen waren. Das war notwendig: Jene Ärzte versäumten es zum damaligen Zeitpunkt nämlich durchaus häufig, Totenscheine für verstorbene Soldaten auszustellen oder diese an die entsprechenden staatlichen Stellen weiterzuleiten. ${ }^{11}$

Auch Jahrzehnte später, als Großbritannien im Verbund mit Frankreich und dem Osmanischen Reich Krieg auf der Krim gegen Russland führte (1853-1856), hatte sich an diesen Problemen wenig geändert. Allerdings

7 Für das Völkerrecht vgl. Mégret 2006, 265-317.

8 Hartmann 2011.

9 Vgl. u.a. Lin 2009, 99-117, 100ff.; Sammlung einzelner Vorschriften, Dienstanweisungen und sonstiger Ausarbeitungen über die Verwaltung der Lazarette bei der Königl. Preußischen Armee (2. in einigen Punkten berichtigte Auflage), Berlin 1815, $283 \mathrm{f}$.

10 Vgl. Lin 2009, 107; Sammlung, 127f., 135f., 191f., 290; Faust 2003, $103 \mathrm{ff}$.

11 Vgl. Sammlung, 283f., 291, 298. 
meldeten sich mittlerweile andere Akteure zu Wort, die ein Interesse daran hatten, neue Praxen der Wissensgenese über den Tod von Soldaten zu etablieren. Eine ganze Reihe von Ärzten und sozialreformerischen Philanthropen drängten zunehmend darauf, dass die Chirurgen in den Lazaretten den Tod von Soldaten, ja überhaupt ihre Krankheiten und Verletzungen, exakter zu erfassen hätten, um - und das war für sie das Entscheidende - zuverlässige Daten über die Mortalitätsursachen der Soldaten im Krieg zu gewinnen. Für dieses Anliegen gab es fraglos ein medizinisches Interesse. Das aber schloss ein weiteres, spezifisches Motiv nicht aus: «Economy in lives is the greatest economy to the state», formulierte 1853 der Autor eines Artikels in der prominenten medizinischen Fachzeitschrift The Lancet, um damit auf ein Problem hinzuweisen, dem sowohl von Seiten der Ärzte als auch von Seiten des Staates entgegengewirkt werden müsse: der Tatsache nämlich, dass der Großteil der Soldaten, die während der Kriege starben, ihr Leben nicht etwa auf dem Schlachtfeld, sondern aufgrund von Krankheiten verlor. Ärzte und Philanthropen sahen darin eine unnötige Schwächung der Armee, denn diese Tode, so das Argument, seien bei einer verbesserten medizinischen Ausstattung und Hygiene in der Armee und in den Lazaretten vermeidbar.

Diesen Zusammenhang nachzuweisen, trat unter anderem Florence Nightingale an, die aus diesem Grund eine akribische Listenführung über die gesundheitlichen Schädigungen und Todesfälle im Lazarett praktizierte und statistische Berechnungen anstellte. ${ }^{12}$ Die Listen, Tabellen und Diagramme, die in diesem Kontext entstanden, waren dabei ein Ort, an dem sich sowohl medizinische als auch politische Anliegen einschrieben und kreuzten. Denn die medizinischen Bemühungen, die Todesursachen von Soldaten genauer zu bestimmen, waren eng mit dem Vorhaben verbunden, der Regierung vor Augen zu führen, dass es im Sinne der nationalen Wehrkraft dringend angezeigt war, dieses medizinische Wissen nicht länger zu ignorieren und Reformen einzuleiten, um in der Armee dem Tod durch Krankheit wirkungsvoller entgegentreten zu können.

Dieses Reformanliegen artikulierte sich in ähnlicher Weise in verschiedenen Ländern - und für fremde Territorien, sofern es das Militär imperialer Mächte betraf. Genauer gesagt, galt die Sorge primär den dort eingesetzten europäischen Soldaten, wie sich etwa nach dem indischen Aufstand von $1857 / 58$ zeigte. Wieder war es Nightingale, die in diesem Zusammenhang aktiv wurde und Druck auf die britische Regierung ausübte. Mit Erfolg: Denn bereits ein Jahr nach dem Aufstand setzte die britische Regierung in London 
die Royal Commission into the Sanitary States of the Army in India ein - mit Nightingale als Mitglied. Ohne jemals indischen Boden zu betreten, sichtete die Kommission in den darauf folgenden Jahren diverse Informationen und Daten, die britische Ärzte in Indien während der vergangenen Jahre über den Gesundheitszustand der dortigen Soldaten (nach europäischen und indischen getrennt) zusammengetragen hatten. Das Ergebnis war für die Kommission, die mit den Augen der Kolonialmacht auf die, ohnehin schon koloniales Wissen produzierenden, Statistiken schaute, beunruhigend: die Krankheits- und Todesrate der weißen britischen Soldaten war demnach nämlich deutlich höher als diejenige der sepoy, der indischen Soldaten. Für die Kommission lag die Schlussfolgerung deshalb auf der Hand: Um die koloniale Herrschaft nicht zu gefährden, legte sie der Regierung in ihrem Bericht aus dem Jahr 1863 dringend nahe, in British-India die Hygiene in der britischen Armee zu verbessern. ${ }^{13}$

Nimmt man die Kriegszeiten des langen 19. Jahrhunderts in den Blick, zeigt sich, dass die medizinischen Aufzeichnungen aus den Lazaretten aber auch auf anderem Wege zirkulierten, was im Kontext einer Wahrnehmungsgeschichte des Krieges interessant ist. Denn die Listen, die Zeitungen veröffentlichten, um der Öffentlichkeit Mitteilung über verstorbene Soldaten zu machen, veränderten sich im Verlauf des 19. Jahrhunderts beträchtlich. Diese Listen wurden nicht nur immer länger (ohne dass dies mit der realen Entwicklung der Kriegstoten übereinstimmte); sie wurden auch immer differenzierter. Waren sie während der Napoleonischen Kriege noch darauf beschränkt, die Namen der verstorbenen Generale zu nennen, machten sie während des Krimkrieges nicht nur Meldung von den Toten, sondern auch von Verletzten, differenziert nach «gefährlich Verletzten», «schwer Verletzten» und «leicht Verletzten»- allerdings immer noch beschränkt auf die Namen der Offiziere. ${ }^{14}$ Während des so genannten Zweiten Burenkrieges (1899-1902) erfassten die Mittelungen dann auch die Namen anderer Soldaten, mit weitaus präziseren Angaben darüber, welche Erkrankung oder Verletzung zum Tod des Soldaten geführt hatte. ${ }^{15}$ Zumindest in den jeweiligen kriegführenden Ländern der sogenannten «zivilisierten» Welt hatten die verletzten und getöteten Soldaten in den Printmedien damit eine bis dahin

13 Prakash 1999, 133; Arnold 1993, 67-74. Differenziertere Ergebnisse und abweichende Interpretationen von britischen Ärzten, die in Indien tätig waren, lagen allerdings durchaus vor, wurden von der Kommission aber ebenso ignoriert wie deren Empfehlungen. Diese Ärzte verwiesen nämlich auf bemerkenswerte Differenzen im Hinblick auf unterschiedliche Krankheiten und führten als Erklärung therapeutische Veränderungen an. Sie empfahlen deshalb, nicht bei der Prophylaxe, sondern bei den Therapien anzusetzen, um die Situation zu verbessern.

14 Beispielhaft dafür: The Times, 13.9.1855, 6.

15 Beispielhaft dafür: The Times, 10.12.1901. 
nicht vorhandene, öffentliche Sichtbarkeit erreicht. Die systematische Sammlung medizinischer Daten in den Lazaretten war dafür nicht die einzige, aber eine wichtige Voraussetzung.

Das trifft im Übrigen in ähnlicher Weise auf die Daten zu, die Eingang in die Militärstatistiken verschiedener europäischer Länder fanden. Seit Mitte der 1860er Jahre präsentierten diese immer reichhaltigere und differenziertere Übersichten und Tabellen, gerade auch im Hinblick auf die statistische Präsentation der Mortalitätsursachen im Krieg. Den Beschlüssen des Internationalen Militärstatistischen Kongresses in Berlin 1863 lässt sich entnehmen, dass die Militärstatistiker aufgrund der statistisch ablesbaren Tatsache, dass die Armeen vor allem durch Krankheiten dezimiert wurden, unbedingten Handlungsbedarf sahen. Die Statistiker sahen sich selbst dabei als die Lieferanten der statistischen Beweise, die den Regierungen vor Augen führen sollten, dass es gelte, den Ursachen für diesen Missstand nachzugehen. Dass «Krankheiten den Heeren oft größere Niederlagen bereiteten als die Angriffe des Feindes», war, wie hier und andernorts deutlich gesagt wurde, nicht akzeptabel. Wege mussten gefunden werden, damit die «Regierung auch ihre Pflicht» erfüllen könne, «die ihrer Sorgen anvertrauten Personen für den Zweck der Vertheidigung des Vaterlandes brauchbar und kräftig zu erhalten». ${ }^{16}$

\section{Experimentieren und regulieren. Die «Zivilisierung» des Krieges}

Dieser wiederholt erhobenen Forderung nach der Erhaltung der Wehrkraft widersprach nicht, dass seit den 1860er Jahren die Sorge um die Gesundheit der Soldaten mit der Idee verknüpft war, den Krieg zu «humanisieren». Das beinhaltete Diverses, ganz zentral aber die Vorstellung, dass Maßnahmen zu entwickeln seien, um die Gefährdung des Lebens von Soldaten im Krieg zu verringern. Dazu bedurfte es medizinischen Wissens. Gemeint ist damit nicht bloß, dass Ärzte während des Krieges vonnöten waren, um erkrankte und verletzte Soldaten zu behandeln. Vielmehr soll an dieser Stelle der Blick darauf gelenkt werden, dass die Medizin, darunter auch die Chirurgie, in verschiedenen europäischen Ländern auch in Friedenszeiten damit befasst war, Wissen darüber herzustellen, welche Verwundungen durch welche Waffen in zukünftigen Kriegen zu erwarten waren, und welche Aussicht es gab, diese Verletzungen zu behandeln.

16 Vgl. Beschlüsse, 41; Zur zeitgenössischen Wahrnehmung, dass Krankheiten den Armeen viel gefährlicher würden als Schlachten u.a. Außerordentliche Beilage zur Allgemeinen Zeitung, Nr. 307, 3.11.1867. 
Die Methoden, die Ärzte dazu anwandten, waren unterschiedlich. Sie notierten ihre Beobachtungen aus Kriegen, erstellten Tabellen aus ihren Daten, setzten diese mit Befunden von Kollegen aus anderen, auch ganz fernen Ländern in Beziehung. ${ }^{17}$ So erwartete der Berliner Chirurg Johannes Gaupp im Jahr 1896 bereits «mit Spannung» den Sanitätsbericht der Japaner über den im Vorjahr erst beendeten japanisch-chinesischen Krieg, zumal er den Chirurgen Japans zusprach, «auf dem Gebiete der Kriegschirurgie Bedeutendes geleistet» zu haben. ${ }^{18}$ Einige Jahre später war es so weit: Die Zahlen aus jenem Krieg über Todesfälle und alle Art von Verletzungen lagen vor; sie konnten sogar bereits mit entsprechenden Zahlen aus dem spanischamerikanischen Krieg von 1898 und dem südafrikanischen Krieg (18981902) verglichen werden. ${ }^{19}$

Ärzte nutzen zu Friedenszeiten aber auch gezielt Experimente. Diese dienten dazu, Wissen zu produzieren, das auf militärische Situationen übertragen werden könne, etwa um Kriegsverläufe abschätzbar zu machen. $\mathrm{Zu}$ diesen Experimenten gehörte, Tieren Schusswunden zuzufügen, um anhand der Schusskanäle und Knochenzertrümmerungen die Geschosswirkung von Projektilen mit unterschiedlichem Kaliber zu testen. So ließ sich etwa aus Sicht des Schweizer Chirurgen Hermann Demme nach entsprechenden Schussversuchen an Pferden zu Beginn der 1860er Jahre nicht mehr behaupten, «dass das kleinere Caliber für den Zweck der Kampfunfähigmachung weniger tauglich sei als das grosse». Entsprechend erschien ihm die vom schweizerischen Bundesrat letztlich getroffene Entscheidung, das kleinere Kaliber im Militär zu verwenden, nach dem «gegenwärtigen Stand unseres Wissens» vollkommen gerechtfertigt. ${ }^{20}$

In den nachfolgenden Jahrzehnten forderte die Entwicklung der Waffentechnik Chirurgen in unterschiedlichen europäischen Ländern beständig zu neuen Experimenten an Leichen, Tieren oder präparierten Objekten

17 So zog der Schweizer Hermann Demme, aufbauend auf den zur Verfügung stehenden Aufzeichnungen über den zweiten italienischen Unabhängigkeitskrieg 1859, die Schlussfolgerung, dass die weitaus größte Zahl der Verletzten in den Lazaretten Schusswunden erlitten hatte. Nur der kleinere Teil war auf Säbel und Bajonett zurückzuführen. Die meisten Soldaten waren dieser Art von Verwundungen nämlich bereits auf dem Schlachtfeld erlegen. Vgl. etwa Demme 1861 (Ausg. 1863), 1ff. Ein vehementer Verfechter des statistischen Wegs war in diesem Zusammenhang auch Ludwig Mayer, wie sich etwa in seiner Studie Kriegschirurgische Mittheilungen aus den Jahren 1870/71, 1873, 35-106, 36f., zeigt. Er verstand seine Aufzeichnungen als Beitrag zur Erstellung einer Gesamtstatistik des Krieges. Diese sei nicht nur notwendig, um aussagekräftige Ergebnisse über die «Fortschritte der Wissenschaft und Humanität» zu erhalten, sondern auch, um Anhaltspunkte für zukünftiges Handeln im Krieg zu gewinnen.

18 Gaupp 1896, 34.

19 Vgl. etwa Stabsarzt Dr. Hildebrandt 1902, 10-36.

20 Demme 1861, 12ff., die Zitate 14 u. 15. 
heraus. In den medizinischen Abhandlungen tritt dabei deutlich zutage, dass die Ergebnisse dieser Experimente dafür herangezogen wurden, die Entwicklung oder eben auch das Verbot bestimmter Waffen zu begründen. Dafür stehen beispielhaft die Experimente des eingangs bereits erwähnten Schweizer Chirurgen Theodor Kocher. Seine Schlussfolgerungen, die er aus diversen Experimenten und Messungen gezogen hatte, gingen in der Schweiz Anfang der 1880er Jahre in die Entwicklung eines Stahlmantelgeschosses ein. ${ }^{21}$ Ein Jahrzehnt später erläuterte Kocher auf dem internationalen Ärztekongress in Rom die Ergebnisse seiner Experimente und plädierte für eine generelle «Verbesserung der Geschosse vom Standpunkte der Humanität». Seine Forderungen beinhalteten, das Kaliber zu reduzieren, ein härteres Geschossmaterial zu verwenden und das vordere Ende des Geschosses zuzuspitzen. Zumindest eine Reihe von Verletzungen fiel dann deutlich geringer aus, argumentierte er, wie auch ihre Behandlung komplikationsloser verlaufe. ${ }^{22}$

Eine solche Argumentation hatte im Rahmen der vielfach eingeforderten Humanisierung des Krieges Gewicht. Nicht nur das: Wie die Debatten um die so genannten Dum-Dum-Geschosse zeigen, die von der britischen Armee erstmals im Jahr 1895 in Indien eingesetzt worden waren, ließ sich damit Politik betreiben. Am ersichtlichsten wurde das im Vorfeld und im Verlauf der ersten Friedenskonferenz von Den Haag 1899. Auf der Grundlage von Experimenten hatte unter anderem der deutsche Chirurg Paul von Bruns argumentiert, dass diese Geschosse unverhältnismäßig große Wunden verursachten und es sich somit um ein inhumanes Kriegsgeschoss handle. ${ }^{23}$ Seine Intervention stieß in einer ganzen Reihe europäischer Staaten auf Gehör. Ausschlaggebend dafür war allerdings weniger, wie die Debatten rund um die Dum-Dum-Geschosse nahelegen, dass deren Verwendung generell auf Kritik stieß. Für das Argument der Briten, die vorbrachten, die ansonsten eingesetzten «humaneren» Projektile hätten sich im Kampf gegen die «Wilden» als unzureichend erwiesen, ${ }^{24}$ zeigten sich viele Vertreter der «zivilisierten» Welt aufgeschlossen. Dafür gibt es Beispiele nicht nur unter den Regierungsvertretern, sondern auch aus den Reihen der Ärzte (kaum erstaunlich, da in der Medizin seinerzeit die Annahme verbreitet war, die «Wilden» seien weniger schmerzempfindlich als die weißen Europäer $)^{25}$ sowie der Juristen. Die Erzählung der Briten über die «Wilden», die trotz

21 Vgl. Fackler u. Dougherty, Kocher and the Scientific Foundation of Wound Ballistics 1991, $153-160$.

22 Vgl. u.a. Kocher 1895, 320-325.

23 Vgl. u.a. von Bruns 1899, 602-607.

24 Spiers 1975, 3-14, 7.

25 Joanna Bourke 2014, 301-319. 
vielfacher Schussverletzungen einfach weiterstürmten und kaum wehrunfähig zu machen waren, stellten viele, wie es scheint, nicht in Frage. Jedenfalls ist bemerkenswert, dass sich diese Geschichten in anderen Texten in vielfacher Nacherzählung wiederfinden. ${ }^{26}$ Insofern waren die entscheidenden Argumente, die für ein Verbot des Dum-Dum-Geschosses sprachen, andere: Es galt, erstens, auszuschließen, dass europäische Staaten, die über derartige Geschosse verfügten, diese auch im Krieg gegen andere «zivilisierte» Staaten einsetzten; und es galt, zweitens, zu vermeiden, dass es den «Aufständischen» in den Kolonien gelingen könnte, dieser Geschosse habhaft zu werden, um sie dann selber im Kampf gegen die Kolonialmächte zu verwenden. Um Letzteres auszuschließen, bedurfte es eines allgemein gültigen Verbots von Explosivgeschossen. Auf der ersten Friedenskonferenz in Den Haag 1899, die aus Sicht des liberalen Staat- und Völkerrechtlers Franz von Liszt «einen wichtigen Fortschritt in der Humanisierung des Krieges» bedeutete, ${ }^{27}$ kamen die dortigen Regierungsvertreter mehrheitlich überein, solche Geschosse zu verurteilen. ${ }^{28}$ Die Vertreter Großbritanniens unterzeichneten nicht; aus ihrer Sicht war der Einsatz dieser Projektile in den Afrikanischen und Asiatischen Kolonien unverzichtbar.

Britischen Chirurgen, die sich in der Folgezeit darum bemühten, mittels weiterer Versuche dem schlechten Ruf der Dum-Dum-Geschosse als inhumane Waffe entgegenzuwirken, war kaum Erfolg beschieden. ${ }^{29}$ Ihre Bemühungen, mithilfe von Zeichnungen die Auswirkungen unterschiedlicher Geschosse im menschlichen Körper darzustellen, unterstrichen bestenfalls, dass es nicht allein die Dum-Dum Geschosse, sondern auch andere Projektile waren, die dramatische Wunden im Körper eines Menschen verursachten. Fotografien von Schädeln, auf die geschossen worden war, ließen sich wiederum als ein Beleg dafür lesen, dass die Dum-Dum-Geschosse eine weitaus größere Zerstörungskraft als andere Geschosse besaßen. ${ }^{30}$ Mit dem Beschluss der Haager Ordnung stand somit fest, dass jeder, der von dieser Waffe Gebrauch machte, mit dem Vorwurf rechnen musste, einen «inhumanen» Krieg zu führen, vielleicht sogar ein «unzivilisierter» Gegner zu sein. In den Augen des britischen Chirurgen Alex Ogston hatten die europäischen Mächte, darunter namentlich Deutschland, Russland, Frankreich, Bulgarien,

26 Vgl. u.a. Meurer 1907, 508.

27 von Liszt ${ }^{10} 1915,32$.

28 In der Haager Landkriegsordnung von 1907 folgte - allerdings ohne weitere Spezifizierung - das Verbot all jener Waffen und Geschosse, die geeignet seien, «unnötiges Leiden zu verursachen». Abkommen betreffend die Gesetze und Gebräuche des Landkrieges, abgeschlossen in Den Haag am 18. Oktober 1907, Art. 23.

29 Vgl. u.a. Ogston 1899, 278-281.

30 Vgl. etwa Keith u. Rigby 1899, 1499-1507. 
bereits durch ihre Haltung gegen den Einsatz explosiver Geschosse versucht, Großbritannien als «anti-humanitär» und «barbarisch» zu brandmarken. ${ }^{31}$

Tatsächlich blieben in den nachfolgenden Kriegen Anschuldigungen an den Kriegsgegner nicht aus, Dum-Dum-Geschosse im Kampf eingesetzt und sich damit den Mitteln einer «unzivilisierten», weil das Völkerrecht verletzenden, Kriegführung bedient zu haben. Industriell gefertigte Projektile dieser Art wurden zwar offenbar nicht eingesetzt, doch ist nicht auszuschließen, dass Soldaten die Projektile selber bearbeiteten, um eine vergleichsweise schädigende Wirkung zu erzielen. In dem Ausmaß, wie von den Kriegsparteien behauptet, wurden Dum-Dum-Geschosse aber nicht verwendet. Vielmehr handelte es sich in vielen Fällen um falsche Informationen, die gezielt verbreitet wurden, um im In- und Ausland gegen den Kriegsgegner zu mobilisieren. Letzteres ist für den südafrikanischen Krieg von 1899/1902 bekannt, ${ }^{32}$ und lässt sich auch für den Ersten Weltkrieg hinreichend nachweisen. ${ }^{33}$

Die Behauptung, dass der Kriegsgegner durch den Einsatz von DumDum-Geschossen ein «barbarisches» Verhalten an den Tag gelegt hätte, war allerdings nur ein Element, das die Propagandaschlacht des Ersten Weltkriegs nährte, und es war gegenüber den Anschuldigungen an die Deutschen, in Frankreich und Belgien Zivilisten ermordet zu haben, gewiss auch ein nachrangiges. Dennoch trifft insgesamt zu, dass die völkerrechtlichen Regelungen der beiden Haager Konferenzen eine zentrale Voraussetzung dafür waren, dass der Erste Weltkrieg zu einem derartigen Propagandakrieg werden konnte. ${ }^{34}$ Dabei handelte es sich zumindest zum Teil um völkerrechtliche Bestimmungen, die - man denke auch an den gesamten Komplex der Regelungen zur Versorgung der Verwundeten - mit dem Feld der Medizin eng verknüpft waren. Diese Verschränkung nahm auch in den darauf folgenden Jahrzehnten nicht ab, im Gegenteil: Denn über eines waren sich Viele schnell im Klaren: Ob die wechselseitigen Beschuldigungen der jeweiligen Kriegsgegner, völkerrechtswidrige Grausamkeiten oder Verbrechen begangen zu haben, substanziell gerechtfertigt waren, ließ sich nicht allein auf der Grundlage eines solchen Vorwurfs entscheiden. Die Anschuldigungen bedurften der Überprüfung. Die internationale Kommission, die im Jahr 1913 im Auftrag des Carnegie Endowment for International Peace eingesetzt wurde, um mögliche Verstöße gegen das Völkerrecht während des Balkankrieges abzuklären, kann als ein Vorbote späterer Kommissionen betrachtet werden, die zu diesem Zweck auf das medizinische Wissen der Forensiker zurückgrif-

31 Ogston 1899, 278.

32 Vgl. u.a. Kuitenbrouwer 2012, 181, 206f.; Geppert, 2007, 132.

33 Vgl. u.a. Pilford 1990, 98-109.

34 In diesem Sinne auch af Jochnick u. Normand 1994, 49-95, 77.

Gesnerus 72 (2015) 
fen. ${ }^{35}$ Bis diese allerdings systematisch in die offizielle Aufklärung von Völkerrechts- und Menschenrechtsverbrechen einbezogen wurden, sollte es noch mehrere Jahrzehnte lange dauern. ${ }^{36}$

\section{Schluss}

Auch heute noch bedarf das Völkerrecht medizinischen Wissens, das gleichzeitig allerdings das Potenzial besitzt, völkerrechtliche Verbote zu umgehen, indem es für die Entwicklung immer perfiderer Waffen nutzbar gemacht wird. Medizin kann damit dem Recht dienen - es kann aber auch gegen das Recht in Stellung gebracht werden. Insofern kann medizinisches Wissen durchaus dazu beitragen, die Leiden im Krieg zu vermindern; es kann aber auch Kriegsgewalt legitimieren, indem etwa der medizinische Nachweis von Verbrechen an Zivilisten für die Rechtfertigung weiterer Gewalteinsätze gegen den Gegner verwendet wird; ebenso kann die Medizin auch Recht unterlaufen, indem etwa medizinisches Wissen dazu herangezogen wird, immer perfidere Kampfstoffe zu erfinden. Ein eindeutiges Verhältnis zwischen Medizin, Recht und Krieg gibt es bis heute nicht.

Bereits in den Jahrzehnten vor Beginn des Ersten Weltkriegs zeichneten die Bemühungen der Chirurgen, die Auswirkungen des Krieges auf den Menschen mithilfe von Daten, Experimenten und Fotografien präziser bestimmen zu können, ein doppeltes Bild des Krieges: Einerseits trugen die Datensammlungen dazu bei, ein anderes Wissen über die Anzahl verletzter und getöteter Soldaten zu erzeugen, die zumindest in den jeweiligen kriegführenden Ländern in den Printmedien eine bis dahin nicht vorhandene, öffentliche Sichtbarkeit erhielten. Andererseits nährten sie unter vielen Chirurgen die Vorstellung, dass die Kriege - die als unvermeidlich galten in ihren Folgen für die Soldaten zunehmend kalkulierbarer, wenn nicht gar beherrschbarer sein könnten. Um die Jahrhundertwende schien das prognostizierbar. Immerhin erachteten Viele das «modern[e] Stahlmantelgeschoss» doch als «humaner», und dachten Gleiches von der «ärztlichen Behandlung», die Verletzten aufgrund veränderter Kenntnisse in der Wundbehandlung «viel größere Chancen» geben würde, mit dem Leben davonzukommen als bislang. ${ }^{37}$

35 Carnegie Endowment for International Peace, Report of the International Commission to Inquire into the Causes and Conduct of the Balkan Wars, Washington 1914.

36 Vorstöße in diese Richtung hatte es allerdings schon nach dem Ersten Weltkrieg gegeben. Vgl. dazu Lewis 2014, 90-93.

37 Skeptischer gegenüber der Annahme eines «humaneren» Krieges: Dilger u. Meyer 1914, 225-379, Zit. 241. 
Als der Erste Weltkrieg begann, zeigte sich relativ schnell, dass diese Zuversicht vollkommen fehl am Platz gewesen war. Stattdessen machten in diesem Krieg zahlreiche Chirurgen eine Erfahrung, die andere Kollegen vor ihnen in früheren Kriegen auch gemacht hatten: Ihre vormaligen Prognosen über die Auswirkungen eines zukünftigen Krieges trafen nie zu, wie sich auch das chirurgische Wissen der Friedenszeit nicht einfach auf die Situation eines Krieges übertragen ließ. Die fachwissenschaftlichen Studien, in denen Chirurgen ihre eigens gemachten Beobachtungen darüber aufzeichneten, wie sich im Krieg Verletzungen entwickelten und chirurgische Eingriffe verliefen, waren sogar wenig dazu geeignet, auf ihnen eine Fortschrittsgeschichte der Medizin aufzubauen. In jedem Krieg, so zeigten diese, stand chirurgisches Wissen auf den Prüfstand: Chirurgen stießen an absolute Grenzen ihrer bislang erlernten therapeutischen Fähigkeiten; in der kriegschirurgischen Tätigkeit stelle sich oftmals sogar, hieß es, «Ratlosigkeit» ein. ${ }^{38}$ Auf Kriege traf zu, was ein Schweizer Chirurg zu Beginn der 1860er Jahre formulierte: Sie waren in vieler Hinsicht eine «Hypothesenschmiede». ${ }^{39} \mathrm{Wie}$ derum war es das Experiment, das in dieser Situation zum Einsatz kam. Denn die einen fanden sich in einer Lage wieder, in der die vorherrschenden chirurgischen Lehrsätze versagten und in der zur Rettung eines Verletzten oder Erkrankten nichts anderes als das Experimentieren übrig blieb. Aus diesem Grund kamen auch chirurgische Praktiken wieder zum Einsatz, die eigentlich längst als überholt galten. ${ }^{40}$ Andere wiederum sahen den Krieg ohnehin unter dem Gesichtspunkt, dass sich in dieser Situation Erfahrungen sammeln ließen, die dazu beitrugen, chirurgisches Wissen - auch zum Nutzen in der zivilen Medizin - zu erweitern. Dies war zumindest einer der erklärten Gründe, die unter anderem die Schweizer Chirurgen Eduard Stierlin und Adolf Vischer dazu veranlassten, als Ärzte am Balkankrieg 1912/13 teilzunehmen..$^{41}$ Sie waren mitnichten die einzigen Chirurgen, die ihre Reise in die fremde Kriegsregion angetreten hatten, um medizinische Erfahrungen zu sammeln. Die Möglichkeit dafür boten den Ärzten die nationalen RotKreuz-Gesellschaften, die, nach Vermittlung des Internationalen Komitees vom Roten Kreuz, Ärzte zur Unterstützung in das Kriegsgebiet beziehungsweise direkt an die kriegführenden Parteien entsandten. Während des Balkankrieges soll sich die Zahl der ausländischen Ärzte, die über ausländische

38 Dilger u. Meyer 1914, 225.

39 Demme 1861, 185.

40 Für den Ersten Weltkrieg gilt das für den Bereich der Wundbehandlung und der Amputationen. Beispiele dazu u.a. in: Kocher 1915.

41 Stierlin u. Vischer 1914, 311-374. Demme, 1861, sprach unumwunden aus, dass es zur Prüfung antiseptischer Mittel nahe gelegen habe, «den Kriegsschauplatz in Italien zu weiteren Experimenten zu benutzen» (Zit. 24). 
Rot-Kreuz-Gesellschaften zur medizinischen Unterstützung entsandt wurden, auf 179 belaufen haben..$^{42}$ Diese kamen aus der Schweiz ${ }^{43}$ und aus Deutschland ${ }^{44}$ aus Großbritannien ${ }^{45}$ und Österreich-Ungarn ${ }^{46}$ oder auch aus Russland. ${ }^{47}$ Ihr deklarierter Auftrag war gemäß den Richtlinien des Internationalen Komitees vom Roten Kreuz ein humanitärer, und die medizinische Hilfe, die die Ärzte im Kriegsgebiet leisteten, für jeden Verwundeten, dem dadurch geholfen werden konnte, zweifellos wertvoll. Doch das war nur ein Effekt dieses Einsatzes. Ein anderer - und durchaus von den nationalen Rot-Kreuz Gesellschaften beabsichtigter - war, die eigenen Ärzte möglichst gut auf eine Situation vorzubereiten, in der das eigene Land selber in einen Krieg verwickelt sein würde. ${ }^{48}$ Bis in die Zeit des Kalten Krieges hinein sollte sich daran nichts Grundlegendes ändern. Die Institution des Internationalen Komitees vom Roten Kreuz war die Plattform, über die sich dieses Anliegen seit den 1860er Jahren bewerkstelligen ließ. Das IKRK stellt seither gewissermaßen den Inbegriff der Verschränkung zwischen Medizin, Recht und dem Anliegen, den Krieg zu «zivilisieren», dar. Was dies jedoch konkret bedeutete, war nicht stabil; es ist nicht zuletzt die Veränderbarkeit medizinischen Wissens, das die Auswirkungen der Kriege und des Völkerrechts bis heute in Fluss hält. ${ }^{49}$.

\section{Bibliographie}

af Jochnick, Chris/Roger Normand, «The Legitimation of Violence: A Critical History of the Laws of War», Harvard International Law Journal 35 (1994) 49-95

Arnold, David, Colonizing the Body. State Medicine and Epidemic Disease in Nineteenth Century India (Berkeley 1993)

Außerordentliche Beilage zur Allgemeinen Zeitung, Nr. 307, 3.11.1867

Berger, Silvia, Bakterien in Krieg und Frieden. Eine Geschichte der medizinischen Bakteriologie in Deutschland 1890-1933 (Göttingen 2009)

Best, Geoffrey, Humanity in Warfare (London 1980)

Boeckh, Katrin, Von den Balkankriegen zum Ersten Weltkrieg, Berlin 1996.

Bourke, Joanna, «Pain Sensitivity: An Unnatural History from 1800 to 1965», Journal of Medical Humanities 35 (2014) 301-319

42 Despot 2012, 186.

43 Draenert 2011.

44 Boeckh 1996, 209.

45 Boeckh 1996, 190.

46 Flamm 2012, 132-144.

47 Despot 2012, 186.

48 Vgl. Flamm 2012, 135.

49 Diese Thematik ist Teil eines von mir gegenwärtig verfolgten größeren Forschungsprojektes. 
Carnegie Endowment for International Peace, Report of the International Commission to Inquire into the Causes and Conduct of the Balkan Wars (Washington 1914)

Demme, Hermann, Militär-Chirurgische Studien in den italienischen Lazarethen von 1859, Bd. 1 (Würzburg 1861; Ausg. 1863)

Despot, Igor, The Balkan Wars in the Eyes of the Warring Parties. Perceptions and Interpretations (Bloomington/In. 2012)

Dilger, Anton/Arthur W. Meyer, «Kriegschirurgische Erfahrungen aus den beiden Balkankriegen 1912/13», Deutsche Zeitschrift für Chirurgie 127 (1914) 225-379

Draenert, Marcelin Oliver, Kriegschirurgie und Kriegsorthopädie in der Schweiz zur Zeit des Ersten Weltkriegs (Diss. phil. Universität Heidelberg 2011)

Dülffer, Jost, «Regeln im Krieg? Kriegsverbrechen und die Haager Friedenskonferenzen», in: Marc Frey/Jost Dülffer (Hrsg.), Frieden stiften. Deeskalations- und Friedenspolitik im 20. Jahrhundert (Köln 2008) 77-88

Eckardt, Wolfgang U., «Der größte Versuch, den die Einbildungskraft ersinnen kann - Der Krieg als hygienisch-bakteriologisches Laboratorium und Erfahrungsfeld», in: Wolfgang Eckardt/Christoph Gradmann (Hrsg.), Die Medizin und der Erste Weltkrieg (Herbolzheim 22003) 299-320

Fackler, Martin L./Paul J. Dougherty, Theodor Kocher and the Scientific Foundation of Wound Ballistics, Surgery, Gynecology \& Obstetrics 172 (1991) 153-160

Faust, Drew Gilpin, This Republic of Suffering. Death and the American Civil War (New York 2003)

Flamm, Heinz, «Das Österreichische Rote Kreuz und österreichische Bakteriologen in den Balkankriegen 1912/13», Wiener Medizinische Wochenschrift 162 (2012) 132-144

Gaupp, Johannes, Über die Grösse und Form des Ein- und Ausschusses (Berlin 1896)

Geppert, Dominik, Pressekriege: Öffentlichkeit und Diplomatie in deutsch-britischen Beziehungen (1896-1912) (London 2007)

Hartmann, Heinrich, Der Volkskörper bei der Musterung. Militärstatistik und Demographie in Europa vor dem Ersten Weltkrieg (Göttingen 2011)

Hildebrandt, Stabsarzt Dr., «Die Prognose der Schussverletzungen im Felde», Deutsche Zeitschrift für Chirurgie 67 (1902) 10-36

Hutchinson, John, Champions of Charity. War and the Rise of the Red Cross (Boulder, Col. 1997)

Keith, Arthur/M. Hugh Rigby, «Modern Military Bullets: A Study of Their Destructive Effects», The Lancet (2.12.1899) 1499-1507

Klencke, Hermann, Über die grosse Sterblichkeit in stehenden Heeren und deren Ursachen (Quedlinburg 1839)

Kocher, Theodor, «Die Verbesserung der Geschosse vom Standpunkt der Humanität», Atti Dell'VI Congresso Medico Internazionale, Roma, 29 Marzo - 5 Aprile 1894, Vol. I (Turin 1895) 320-325

Kocher, Theodor, «Eindrücke aus deutschen Kriegslazaretten», CorrespondenzBlatt für Schweizer Ärzte 45 (1915) 449-479

Kuitenbrouwer, Vincent, War of Words: Dutch Pro-Boer Propaganda and the South African War (1899-1902) (Amsterdam 2012)

Lewis, Mark, The Birth of a New Justice. The Internationalization of Crime and Punishment, 1919-1950 (Oxford 2014) 
Lin, Patricia Y.C.E., «Caring for the Nation's Families: British Soldiers' and Sailors' Families and the State, 1793-1815», in: Alan Forrest u.a. (Hrsg.), Soldiers, Citizens and Civilians: Experiences and Perceptions of the Revolutionary and Napoleonic Wars, 1790-1820 (New York 2009) 99-117

Linton, Derek S., «The Obscure Object of Knowledge: German Military Medicine Confronts Gas Gangrene During World War I», Bull. Hist. Med. 74 (2000) 291-316

Logan, Deborah Anna/Harriet Martineau, Victorian Imperialism, and the Civilizing Mission (Farnham, Surrey 2009)

Magnello, M. Eileen, «The Passionate Statistitian», in: Nelson Siobian/Anne Marie Rafferty (eds.), Notes on Nightingale. The Influence and Legacy of a Nursing Icon, (New York 2010) 115-129

Mayer, Ludwig, «Kriegschirurgische Mittheilungen aus den Jahren 1870/71», Deutsche Zeitschrift für Chirurgie 3 (1873) 35-106

McDonald, Lynn, The Women Founders of the Social Sciences (Montreal 1994)

Mégret, Frédéric, «From 'savages' to 'unlawful combatants': a postcolonial look at international law's 'other'», in: Anne Orford (ed.), International Law and its 'Others' (Cambridge 2006) 265-317

Meurer, Christian, Die Haager Friedenskonferenz (München 1907)

Ogston, Alex, «The Peace Conference and the Dum-Dum Bullet», The British Medical Journal 2 (1899) 278-281

Pilford, Neil, «Controversy over the Use of Dum Dum Bullets in the First World War», Imperial War Museum Review 5 (1990) 98-109

Sammlung einzelner Vorschriften, Dienstanweisungen und sonstiger Ausarbeitungen über die Verwaltung der Lazarethe bei der Königl. Preußischen Armee (2. in einigen Punkten berichtigte Auflage) (Berlin 1815)

Schlich, Thomas, «Asepsis and Bacteriology: A Realignment of Surgery and Laboratory Science», Medical History 56 (2012) 308-334

Segesser, Daniel Marc, Recht statt Rache oder Rache durch Recht? Die Ahndung von Kriegsverbrechen in der internationalen fachwissenschaftlichen Debatte 1872-1945 (Paderborn 2007)

Spiers, Edward M., «The use of the Dum Dum bullet in colonial warfare», The Journal of Imperial and Commonwealth History 4 (1975) 3-14

Stierlin, Eduard/Adolf L. Vischer, «Chirurgische Beobachtungen auf allen Etappen im serbisch-türkischen Kriege 1912-1913», Deutsche Zeitschrift für Chirurgie 131 (1914) 311-374

The Times, 10.12.1901

The Times, 13.9.1855, 6

von Bruns, Paul, «Inhumane Kriegs-Geschosse», Archiv für Klinische Chirurgie 57 (1899) 602-607

von Liszt, Franz, Das Völkerrecht (Berlin $\left.{ }^{10} 1915\right)$

von Schjerning, Otto, Die Tätigkeit und die Erfolge der deutschen Feldärzte im Weltkriege, zugleich Einleitung zu dem 'Handbuch der ärztlichen Erfahrungen im Weltkriege' (Leipzig 1920) 